\title{
Ultrafast and Reproducible Proteomics from Small Amounts of Heart Tissue Enabled by Azo and timsTOF Pro
}

Timothy J. Aballo ${ }^{1,2}$, David S. Roberts ${ }^{3}$, Jake A. Melby ${ }^{3}$, Kevin M. Buck ${ }^{3}$, Kyle A. Brown ${ }^{3,4}$, and Ying $\mathrm{Ge}^{2,3,5^{*}}$

${ }^{1}$ Molecular and Cellular Pharmacology Training Program, University of Wisconsin-Madison, Madison, Wisconsin 53705, USA

${ }^{2}$ Department of Cell and Regenerative Biology, University of Wisconsin-Madison, Madison, Wisconsin 53705, USA

${ }^{3}$ Department of Chemistry, University of Wisconsin-Madison, Madison, Wisconsin 53706, USA.

${ }^{4}$ Department of Surgery, University of Wisconsin-Madison, Madison, Wisconsin, 53706, USA

${ }^{5}$ Human Proteomics Program, School of Medicine and Public Health, University of WisconsinMadison, Madison, Wisconsin 53705, USA.

*Correspondence should be addressed to Y.G. (ying.ge@wisc.edu) 


\begin{abstract}
Global bottom-up mass spectrometry (MS)-based proteomics is widely used for protein identification and quantification to achieve a comprehensive understanding of the composition, structure, and function of the proteome. However, traditional sample preparation methods are timeconsuming, typically including overnight tryptic digestion, extensive sample clean-up to remove MS-incompatible surfactants, and offline sample fractionation to reduce proteome complexity prior to online liquid chromatography-tandem mass spectrometry (LC-MS/MS) analysis. Thus, there is a need for a fast, robust, and reproducible method for protein identification and quantification from complex proteomes. Herein, we developed an ultrafast bottom-up proteomics method enabled by Azo, a photocleavable, MS-compatible surfactant that effectively solubilizes proteins and promotes rapid tryptic digestion, combined with the Bruker timsTOF Pro, which enables deeper proteome coverage through trapped ion mobility spectrometry (TIMS) and parallel accumulation-serial fragmentation (PASEF) of peptides. We applied this method to analyze the complex human cardiac proteome and identified nearly 4,000 protein groups from as little as $1 \mathrm{mg}$ of human heart tissue in a single one-dimensional LC-TIMS-MS/MS run with high reproducibility. Overall, we anticipate this ultrafast, robust, and reproducible bottom-up method empowered by both Azo and the timsTOF Pro will be generally applicable and greatly accelerate the throughput of large-scale quantitative proteomic studies. Raw data are available via the MassIVE repository with identifier MSV000087476.
\end{abstract}

Keywords: photocleavable surfactant, human heart proteomics, quantitative proteomics, sample preparation, bottom-up proteomics 


\section{Introduction}

Global bottom-up mass spectrometry (MS)-based proteomics is widely used for protein identification and quantification to achieve a comprehensive understanding of the composition, structure, and function of the proteome. ${ }^{1-3}$ However, typical bottom-up methods are timeconsuming, involving extraction and solubilization of proteins, reduction and alkylation of disulfide bonds, enzymatic digestion of proteins into peptides, and removal of MS-incompatible surfactants prior to online liquid chromatography (LC)-tandem MS (MS/MS). ${ }^{3-7}$ Surfactants are commonly used during sample preparation to improve protein solubility, especially for membrane proteins; however, most surfactants are MS-incompatible and thus need to be removed before LCMS/MS which further complicates and extends the sample clean-up process. ${ }^{7-12}$

Recently, we developed an anionic, photocleavable surfactant, 4-hexylphenylazosulfonate (referred to as "Azo"), which promotes efficient extraction and solubilization of proteins with similar performance to SDS but can rapidly degrade upon UV exposure. ${ }^{13}{ }^{14}$ Importantly, we have shown the unique capability of Azo as an "all-in-one" MS-compatible surfactant for both top-down and bottom-up proteomics, which enables high-throughput sample handling before MS-analysis. ${ }^{13}$ Azo promotes rapid and robust digestion, even for trypsin-resistant proteins, and thus Azo-aided bottom-up methods have been successfully developed for effective membrane and extracellular matrix proteomics. ${ }^{13,15}$ As enzymatic digestion is a major time-consuming step in bottom-up sample preparation, removing this bottleneck greatly speeds up sample processing time. Nevertheless, the proteome coverage was limited in these Azo-enabled bottom-up proteomics studies using one-dimensional (1D) reversed-phase (RP)LC-MS/MS, in part due to the instrumentation (a standard quadrupole time of flight (Q-TOF) mass spectrometer) used for data acquisition. $^{13,15}$ 
To obtain deeper proteome coverage, multidimensional separation (often involving offline sample fractionation) is employed for the analysis of complex proteomes, which adds another time-consuming step in the bottom-up proteomics workflow. ${ }^{16-20}$ Though there have been developments in online multidimensional LC separation coupled to MS analysis, these current online two-dimensional (2D) LC-MS/MS methods remain technically challenging to implement and can lead to decreased sensitivity. ${ }^{21-23}$ Recently, Bruker interfaced trapped ion mobility spectrometry (TIMS) with the fast scan speeds of Q-TOF mass spectrometers in their timsTOF Pro, an instrument that provides an additional dimension of peptide separation in the gas phase. When the timsTOF Pro is operated in parallel accumulation-serial fragmentation (PASEF) mode, peptide ions that co-elute during LC are accumulated in parallel at specific mobility spaces, and then, in conjunction with rapid quadrupole switching, are serially ejected from the TIMS cell for MS/MS analysis. ${ }^{24,25}$ This process increases sequencing speeds, without a loss in sensitivity, leading to increased proteome sequencing depth. ${ }^{24,25}$

Here, for the first time, we report an ultrafast and reproducible quantitative global bottomup proteomics method with broad proteome coverage enabled by Azo and the timsTOF Pro. We applied this method to identify and reproducibly quantify the proteome from very small amounts of heart tissue. We achieved rapid tryptic digestion in $30 \mathrm{~min}$ and identified nearly 4,000 protein groups in a single 1D LC-TIMS-MS/MS run from just $1 \mathrm{mg}$ of human left ventricular (LV) tissue. We envision this ultrafast, robust, reproducible, and quantitative bottom-up method can be generally applicable and can greatly accelerate the throughput of large-scale quantitative proteomic studies even when samples are limited. 


\section{Materials and Methods}

Chemicals and reagents. 4-hexylphenylazosulfonate (Azo) was synthesized in-house as previously described. ${ }^{13,14}$ All reagents were purchased from Millipore Sigma (St. Louis, MO, USA) and Fisher Scientific (Fair Lawn, NJ, USA) unless noted otherwise. All solutions were prepared with HPLC-grade water (Fisher Scientific).

Sample Preparation. Donor hearts were obtained from the University of Wisconsin Organ Procurement Organization. Tissue collection procedures were approved by Institutional Review Board of the University of Wisconsin-Madison (Study \# 2013-1264). Donor heart tissue was maintained in cardioplegic solution before dissection, and after dissection, the tissue was immediately snap-frozen in liquid nitrogen and stored at $-80^{\circ} \mathrm{C}$.

In a cold room $\left(4{ }^{\circ} \mathrm{C}\right)$, human $\mathrm{LV}$ tissue was quickly cut into $1 \mathrm{mg}, 5 \mathrm{mg}$ and $20 \mathrm{mg}$ pieces then washed twice in 20 tissue volumes $(20 \mu \mathrm{L} / \mathrm{mg}) \mathrm{Mg}^{2+} / \mathrm{Ca}^{2+}$-free DPBS containing 1X HALT protease and phosphatase inhibitor (Thermo Fisher Scientific, Waltham, MA, USA). After washing, tissues were homogenized in 10 tissue volumes $(10 \mu \mathrm{L} / \mathrm{mg})$ of Azo lysis buffer $(0.2 \%$ w/v Azo, $25 \mathrm{mM}$ ammonium bicarbonate, $10 \mathrm{mM}$ L-methionine, $1 \mathrm{mM}$ dithiothreitol (DTT), and 1X HALT protease and phosphatase) using a Teflon pestle (1.5 mL micro-centrifuge tube, flat tip; Thomas Scientific, Swedesboro, NJ, USA). ${ }^{13}$ Samples were diluted to $0.1 \%$ Azo and then homogenized. Samples were centrifuged at $21,100 \mathrm{~g}$ for $30 \mathrm{~min}$ at $4{ }^{\circ} \mathrm{C}$, and the supernatant was transferred to a new tube.

Samples were normalized to $1 \mathrm{mg} / \mathrm{mL}$ protein in $0.1 \%$ Azo by the Bradford assay (BioRad, Hercules, CA, USA, Cat\# 5000006) using bovine serum albumin to generate a standard curve. Disulfide bonds were reduced with $30 \mathrm{mM}$ DTT at $37{ }^{\circ} \mathrm{C}$ for $1 \mathrm{~h}$ and alkylated with $30 \mathrm{mM}$ iodoacetamide in the dark for 45 min. Trypsin Gold (Promega, Madison, WI, USA) was added in 
a protein:protease ratio (wt/wt) of 50:1 and incubated for $30 \mathrm{~min}, 1 \mathrm{~h}$, or $24 \mathrm{~h}$ at $37^{\circ} \mathrm{C}$. Enzymatic activity was quenched by acidifying samples to $\mathrm{pH} 2$ with formic acid (FA) and placing samples on ice. After digestion, Azo was degraded at 305 nm (UVN-57 Handheld UV Lamp; Analytik Jena, Jena, TH, DEU) for $5 \mathrm{~min}$, samples were spun at $21,100 \mathrm{~g}$ at $4{ }^{\circ} \mathrm{C}$ for $15 \mathrm{~min}$, and then desalted using $100 \mu \mathrm{L}$ Pierce C18 tips (Thermo Fisher Scientific) following the manufacturer's specifications to remove Azo degradants and other salts typically present in the digestion. Desalted peptides were dried in a vacuum centrifuge and reconstituted in $0.1 \%$ FA. The peptide concentration was determined by A205 readings using a NanoDrop. Samples were centrifuged at $21,100 \mathrm{~g}$ at $4^{\circ} \mathrm{C}$ for $30 \mathrm{~min}$ prior to analysis.

Bottom-up Data Acquisition. LC-TIMS-MS/MS was carried out using a nanoElute nano-flow ultra-high pressure LC system (Bruker Daltonics, Bremen, Germany) coupled to the timsTOF Pro, a TIMS Q-TOF mass spectrometer (Bruker Daltonics), using a CaptiveSpray nano-electrospray ion source (Bruker Daltonics). For most analyses, $200 \mathrm{ng}$ of peptide digest was loaded on a capillary C18 column ( $25 \mathrm{~cm}$ length, $75 \mu \mathrm{m}$ inner diameter, $1.6 \mu \mathrm{m}$ particle size, $120 \AA$ pore size; IonOpticks, Fitzroy, VIC, AUS). To assess instrument sensitivity with complex heart proteome digest, peptide loading amount was decreased from $200 \mathrm{ng}$ per injection down to $6.25 \mathrm{ng}$. Peptides were separated at $55^{\circ} \mathrm{C}$ using a $120 \mathrm{~min}$ gradient at a flow rate of $400 \mathrm{~nL} / \mathrm{min}$ (mobile phase A (MPA): 0.1\% FA; mobile phase B (MPB): 0.1\% FA in acetonitrile). A stepwise gradient of 2-17\% MPB was applied for $60 \mathrm{~min}$, followed by a step from 17-25\% MPB from $60-90 \mathrm{~min}, 25-37 \%$ MPB from 90-100 min, 37-85\% MPB from 100-110 min, and finished with a wash at 85\% MPB for an additional $10 \mathrm{~min}$ for a total runtime of $120 \mathrm{~min}$ per analysis.

The timsTOF Pro was operated in PASEF mode. ${ }^{24,25}$ Mass spectra for MS and MS/MS scans were recorded between $100-1700 \mathrm{~m} / \mathrm{z}$. Ion mobility resolution was set to $0.60-1.60 \mathrm{~V}^{*} \mathrm{~s} / \mathrm{cm}$ 
over a ramp time of $100 \mathrm{~ms}$. Data-dependent acquisition was performed using 10 PASEF MS/MS scans per cycle with a near $100 \%$ duty cycle. A polygon filter was applied in the $m / z$ and ion mobility space to exclude low $\mathrm{m} / \mathrm{z}$, singly charged ions from PASEF precursor selection. An active exclusion time of 0.4 min was applied to precursors that reached 20,000 intensity units. Collisional energy was ramped stepwise as a function of ion mobility. ${ }^{24}$ Peptide loading was normalized to the total ion chromatograms from $200 \mathrm{ng}$ injections of K562 whole cell lysate standard (Promega).

Data Analysis. MS raw files were processed with MaxQuant ${ }^{26}$ (version 1.6.17.0), MS/MS spectra were matched to in silico derived tryptic peptide fragment mass values from the Uniprot human database UP000005640 (accessed 04 September 2020) and potential contaminants by the Andromeda search engine. ${ }^{27}$ Peptide mass was limited to 8,000 Da with a maximum of two missed cleavages and a minimum length of 7 amino acids. Methionine oxidation and acetylation of protein N-termini were set as variable modifications, whereas carbamidomethylation of cysteine was set as a fixed modification. Initial maximum mass tolerance was set at $70 \mathrm{ppm}$ for precursor ions and $40 \mathrm{ppm}$ for fragment ions similarly as reported previously. ${ }^{28}$ Individual precursor mass tolerances were applied to each peptide spectral match (PSM) by using the default parameters on MaxQuant. Match between runs was enabled with a retention time matching window of 0.7 min and an ion mobility matching window of $0.05 \mathrm{~V}^{*} \mathrm{~s} / \mathrm{cm}^{2}$. Label-free quantification (LFQ) was performed using classic normalization and a minimum ratio count of 2 . A reverse sequence library was generated to control the false discovery rate at less than $1 \%$ for PSMs and protein group identifications.

After MaxQuant processing, data were further analyzed and visualized using Perseus (version 1.6.14.0). ${ }^{29}$ Data were filtered to remove potential contaminants, reverse hits, and protein groups “only identified by site". Protein intensities were $\log _{2}$ transformed, and scatterplots of protein group LFQ intensities were generated between samples and annotated with Pearson 
correlation coefficients. Comparisons of unique protein groups and unique peptides identified by MaxQuant were visualized with Bio Venn. ${ }^{30}$

\section{Results and Discussion}

Azo Enabled Ultrafast Proteomics using the timsTOF Pro. The goal of this study was to develop an ultrafast and reproducible quantitative proteomics method from small amounts of heart tissue using the timsTOF Pro (Figure 1A). Previously we demonstrated that Azo, an in-house developed photocleavable and MS compatible anionic surfactant, effectively solubilized proteins and promoted rapid enzymatic digestion. ${ }^{13}$ However, the reproducibility of these ultrafast digestions for quantitative proteomics had not been demonstrated using the timsTOF Pro for data acquisition. As the heart proteome is highly complex and the timsTOF Pro's enhanced sensitivity enables deep proteome coverage, we set out to test if Azo in combination with the timsTOF Pro could enable ultrafast quantitative proteomics with high reproducibility and high proteome coverage. The timsTOF Pro greatly increases MS/MS sequencing speed through PASEF without sacrificing sensitivity, ${ }^{24,25}$ a mode in which ions that co-elute from the LC column are accumulated in parallel in the TIMS cell, separated into discreet packets based on their TIMS mobility, and then, in conjunction with rapid quadrupole switching, are serially ejected and subjected to MS/MS (Figure 1B). This process substantially increases the number of protein groups and peptides identified from K562 whole cell lysate digest as a reference standard (Supplementary Figure S1). Utilizing PASEF, we were able to identify 6,364 protein groups and 60,159 peptides from three injections of $200 \mathrm{ng}$ of K562 whole cell lysate, compared to only 2,531 protein groups and 15,815 peptides from the same sample with PASEF and TIMS disabled, exemplifying how PASEF greatly expands proteome coverage. 
Using this platform, we first set out to determine if Azo could enable ultrafast enzymatic digestion of the human heart proteome. Previously, we had shown that Azo could be employed to reduce digestion times down to $30 \mathrm{~min}$, but the proteome coverage in this previous study was limited as data acquisition was performed on the Impact II (Bruker), a standard Q-TOF MS, and without any offline sample fractionation. ${ }^{13}$ Therefore, we sought to evaluate if these ultrafast digestions demonstrate reproducibility for quantitative proteomics on the timsTOF Pro, a sensitive instrument that provides much deeper proteome coverage. To do so, we homogenized three replicates of $20 \mathrm{mg}$ of human LV cardiac tissue, a commonly used tissue for heart proteomics, in an Azo-containing buffer to extract and solubilize cardiac proteins. After sample normalization, thiol-containing cysteine residues were reduced and alkylated and then proteins were digested at $37{ }^{\circ} \mathrm{C}$ with trypsin in a $0.1 \%$ (w/v) Azo solution as described previously. ${ }^{13}$ Tryptic digestions were halted after $30 \mathrm{~min}, 1 \mathrm{~h}$, and $24 \mathrm{~h}$ and then prepared for LC-TIMS-MS/MS analysis (Figure 1A). After just 30 min of enzymatic digestion in $0.1 \%$ Azo, the extracted cardiac proteome was nearly completely digested, similar to $24 \mathrm{~h}$ digested samples (Figure 2A). From the data collected by LCTIMS-MS/MS, we observed a high degree of overlap in protein groups and peptides identified among the different digest times (Figure 2B and 2C, Supplementary Table S1). Notably, within three extraction replicates, 4,016 unique protein groups from 36,312 unique peptides were identified after only 30 min of tryptic digest (Figure $2 \mathrm{~B}$ and $2 \mathrm{C}$ ). From these same three extractions, 3,834 protein groups and 37,604 peptides were identified after $1 \mathrm{~h}$ of digestion, and 3,841 protein groups and 37,054 peptides were identified after $24 \mathrm{~h}$ of digestion (Figures $2 \mathrm{~B}$ and 2C). These data indicate that 30 min of enzymatic digestion in $0.1 \%$ Azo sufficiently digests the cardiac proteome, leading to a similar number of protein group and peptide identifications. 
Subsequently, we sought to evaluate the reproducibility of these ultrafast 30 min tryptic digestions, as reproducible sample preparation and quantitation is critical for future biological studies. Extraction replicates from the same piece of tissue after 30 min tryptic digestions were normally distributed and displayed a strong correlation in protein group LFQ intensities (Figure 2D, Supplementary Figures S2 and S3). These data were similar after $1 \mathrm{~h}$ and $24 \mathrm{~h}$ digestions (Figure 2E and 2F, Supplementary Figure S2 and S3), suggesting that 30 min rapid digestion lead to highly reproducible and quantitatively similar protein group and peptide LFQ intensities. Furthermore, protein group LFQ intensities between the different digestion times also displayed similar correlations (Figure 2G-I), indicating that increased digest time does not strongly alter protein group LFQ intensity (Supplementary Figure S4). Our data indicates that with 30 min of Azo-enhanced tryptic digest, proteins are well digested, producing similar protein group and peptide LFQ intensities when compared to $1 \mathrm{~h}$ and $24 \mathrm{~h}$ tryptic digestions.

Reproducible Proteome Analysis from Small Amounts of Tissue. Next, we sought to determine whether this Azo-enabled sample preparation, including ultrafast tryptic digestion, could be applied to small amounts of cardiac tissue. In studies involving limited amounts of tissue, such as those analyzing precious clinical samples or cardiac tissue harvested from small organisms, reducing the amount of tissue required for protein extraction and processing is highly desirable. Using our method, we were able to extract and analyze a similar number of protein groups and peptides from $20 \mathrm{mg}, 5 \mathrm{mg}$ and $1 \mathrm{mg}$ tissue extractions, with 3,047 shared protein groups and 28,945 shared peptides identified among the three tissue extractions (Figure 3A and 3B, Supplementary Figure S5, Supplementary Table S2). Notably, within three extraction replicates from only $1 \mathrm{mg}$ of tissue, we identified 4,114 unique protein groups and 38,319 unique peptides 
(Figure 3A and 3B). We observed a similar number of unique protein groups and peptides from $20 \mathrm{mg}$ (4,279 and 40,276, respectively) and $5 \mathrm{mg}$ (4,049 and 37,367, respectively) tissue extractions.

As the heart proteome is highly complex, many previous global bottom-up heart proteomics studies employed offline peptide fractionation prior to online LC-MS/MS to increase proteome sequencing depth. ${ }^{31-37}$ For example, a global bottom-up proteomics analysis of heart chamber tissue from rhesus monkeys made use of overnight tryptic digestion, isobaric peptide labelling, and offline sample fractionation before analysis with a highly sensitive Orbitrap Fusion (Thermo), which altogether lead to the identification of 1,633 protein groups. ${ }^{36}$ Similarly, a global proteomic profiling of human LV tissue from patients with end-stage dilated cardiomyopathy identified a total of 4,263 protein groups after $15 \mathrm{~h}$ of digestion with Lys-C and trypsin, Sep-Pak desalting, isobaric iTRAQ labeling, and offline $\mathrm{pH}$-based peptide prefractionation prior to online LC-MS/MS. ${ }^{37}$ Moreover, another study by the Mann group identified over 7,000 protein groups from human LV tissue, this was accomplished through extensive offline $\mathrm{pH}$-based fractionation with an automated "loss-less" nano-fractionator, ${ }^{38}$ resulting in 54 total fractions yielding 8 LCMS/MS runs per sample using Q Exactive HF (Thermo). ${ }^{31}$ Here, using our Azo-enabled bottomup proteomics method, we identified nearly 4,000 protein groups in a single 1D LC-TIMS-MS/MS run (Supplementary Figure S6), greatly speeding up sample processing and data acquisition while providing deep proteome coverage. Importantly, our method is high-throughput (30 min total digestion time) and does not require extensive sample preparation, which makes this Azo-enabled method more amenable to large-scale biological studies.

Additionally, extraction replicates from $20 \mathrm{mg}, 5 \mathrm{mg}$, and $1 \mathrm{mg}$ of tissue demonstrated highly reproducible, normally distributed protein group LFQ intensities (Figure 3C-E, 
Supplementary Figure S7 and S8), and protein group LFQ intensities were similar among the different tissue extraction amounts (Figure 3F-H). While there were some differentially extracted protein groups $(\mathrm{p} \leq 0.01)$ among the various tissue amounts (Supplementary Figure S9), these changes are likely due to biological heterogeneity at small scales. Overall, these results indicate that we have developed a robust bottom-up sample processing method that employs ultrafast enzymatic digestion of the human cardiac proteome using Azo and that this method can be scaled down to small tissue amounts while maintaining high proteome coverage and strong reproducibility between extraction replicates for quantitative proteomics.

High Proteome Coverage from Low Amounts of Peptide on the timsTOF Pro. Lastly, we wanted to investigate the sensitivity and reproducibility of the timsTOF Pro when analyzing low amounts of tryptic peptides. From three different extraction replicates from $20 \mathrm{mg}$ of cardiac tissue, we analyzed 200, 100, 50, 25, 12.5 and $6.25 \mathrm{ng}$ of digested peptides by LC-TIMS-MS/MS. From three injections of just $6.25 \mathrm{ng}$ of peptide, we were able to identify 1,532 unique protein groups and 9,503 unique peptides (Figure 4A and 4B, Supplementary Table S3). While this is 32-fold less peptide mass than loaded in $200 \mathrm{ng}$ injections, we identified only 3-fold more protein groups $(4,303)$ and 4-fold more peptides $(40,276)$ in three $200 \mathrm{ng}$ injections, highlighting the timsTOF Pro's remarkable sensitivity. Notably, throughout this injection series, protein group LFQ intensities were normally distributed, and there were strong correlations among $200 \mathrm{ng}$ injections and among $6.25 \mathrm{ng}$ injections, indicating there is strong reproducibility even at low peptide loading amounts (Figure 4C and 4D, Supplementary Figures S10 and S11). In cases where sample amounts are extremely limited, the timsTOF Pro, with its enhanced sensitivity due to its TIMS cell, can 
reproducibly identify a substantial number of protein groups and peptides from very small amounts of tryptic digest.

In summary, we have developed an ultrafast bottom-up method for quantitatively analyzing the human cardiac proteome. Our workflow is quick, employing rapid (30 min) tryptic digestion without sacrificing proteome coverage or reproducibility and can be easily scaled down to small amounts of tissue. We achieve deep proteome coverage, identifying nearly 4,000 protein groups in a single LC-TIMS-MS/MS run from a $1 \mathrm{mg}$ tissue extraction without extensive sample cleanup and fractionation, benefiting from both Azo and the timsTOF Pro. Overall, we anticipate this robust, ultrafast, and reproducible bottom-up method for quantitatively analyzing the human cardiac proteome will be the standard procedure for large-scale proteomic studies to answer an array of biological questions not only in cardiac tissue but also in other tissues with highly complex proteomes.

\section{Associated Content}

\section{Supporting Information}

The Supporting Information is available free of charge at

Supplementary Methods:

SDS-Polyacrylamide Gel Electrophoresis (SDS-PAGE)

Bottom-Up Data Acquisition without TIMS/PASEF

Data Analysis for K562 Whole-Cell Digest Comparison

Supplementary Figures:

Supplementary Figure S1. timsTOF Pro and PASEF enable deep proteome coverage 
Supplementary Figure S2. $\log _{2}$ LFQ protein group intensities for each digestion time are normally distributed.

Supplementary Figure S3. Individual digestion replicates show reproducible $\log _{2}$ LFQ protein intensities

Supplementary Figure S4. Comparison of protein abundance between different tryptic digestion times

Supplementary Figure S5. SDS-PAGE demonstrating reproducible proteome extraction from different tissue extraction amounts

Supplementary Figure S6. Comparison of protein groups identified between different tissue extraction replicates

Supplementary Figure S7. $\log _{2}$ LFQ protein group intensities for each tissue extraction amount are normally distributed

Supplementary Figure S8. Individual replicates from different tissue amounts show reproducible $\log _{2}$ LFQ protein intensities

Supplementary Figure S9. Comparison of proteins extracted between different tissue amounts

Supplementary Figure $S 10$. $\log _{2}$ LFQ protein group intensities are normally distributed at low peptide loading amounts

Supplementary Figure S11. $200 \mathrm{ng}$ and $6.25 \mathrm{ng}$ peptide injection replicates show reproducible $\log _{2} L F Q$ protein intensities

Supplementary Tables:

Supplementary Table S1. Protein groups identified after $24 \mathrm{~h}, 1 \mathrm{~h}$, and 30 min tryptic digestions 
Supplementary Table S2. Protein groups identified from $20 \mathrm{mg}, 5 \mathrm{mg}$, and $1 \mathrm{mg}$ tissue extractions

Supplementary Table S3. Protein groups identified from 200, 100, 50, 25, 12.5, and 6.25 ng peptide injections

\section{Acknowledgements}

Financial support was provided by the National Institutes of Health (NIH) R01 HL096971 (to Y.G.). Y.G. would also like to acknowledge R01 GM117058, GM125085, HL109810 and S10 OD018475. T.JA. would like to acknowledge support from the Training Program in Molecular and Cellular Pharmacology, T32 GM008688-20. J.A.M. would like to acknowledge support from the Training Program in Translational Cardiovascular Science, T32 HL007936-20. K.A.B. would like to acknowledge the Cardiovascular Research Center Training Program in Translational Cardiovascular Science, T32 HL007936-19 and Vascular Surgery Research Training Program grant T32HL110853. We would like to thank Mel Park, Oliver Raether, Guillaume Tremintin, Yue Ju, Conor Mullins, Michael Greig, Gary Kruppa, Paul Speir and Rohan Thakur of Bruker Daltonics for their kind help and provision of the Bruker timsTOF Pro used in this work. Moreover, we would like to acknowledge James Anderson and Carrie Sparks at the University of Wisconsin Organ and Tissue Donation for providing donor hearts. Some aspects of the TOC, Figure 1, and Figure 3 were created using BioRender.com.

\footnotetext{
Abbreviations

MS, mass spectrometry; LC, liquid chromatography; MS/MS, tandem MS; 1D, one-dimensional; RP, reversed-phase; Q-TOF, quadrupole time of flight; 2D, two-dimensional; TIMS, trapped ion mobility; PASEF, parallel-accumulation-serial fragmentation; LV, left ventricular; DTT,
} 
bioRxiv preprint doi: https://doi.org/10.1101/2021.05.24.445470; this version posted May 26, 2021. The copyright holder for this preprint (which

was not certified by peer review) is the author/funder, who has granted bioRxiv a license to display the preprint in perpetuity. It is made available under aCC-BY-NC-ND 4.0 International license.

dithiothreitol; FA, formic acid; MPA, mobile phase A; MPB, mobile phase B; PSM, peptide spectral match; LFQ, label-free quantification 


\section{References}

1. Zhang, Y.; Fonslow, B. R.; Shan, B.; Baek, M.-C.; Yates, J. R., 3rd, Protein analysis by shotgun/bottom-up proteomics. Chemical reviews 2013, 113 (4), 2343-2394.

2. $\quad$ Gillet, L. C.; Leitner, A.; Aebersold, R., Mass Spectrometry Applied to Bottom-Up Proteomics: Entering the High-Throughput Era for Hypothesis Testing. Annual Review of Analytical Chemistry 2016, 9 (1), 449-472.

3. Aebersold, R.; Mann, M., Mass-spectrometric exploration of proteome structure and function. Nature 2016, 537 (7620), 347-355.

4. Weston, L. A.; Bauer, K. M.; Hummon, A. B., Comparison of bottom-up proteomic approaches for LC-MS analysis of complex proteomes. Analytical Methods 2013, 5 (18), 4615-4621.

5. Kuljanin, M.; Dieters-Castator, D. Z.; Hess, D. A.; Postovit, L.-M.; Lajoie, G. A., Comparison of sample preparation techniques for large-scale proteomics. PROTEOMICS 2017, 17 (1-2), 1600337.

6. $\quad$ Zhang, Z.; Wu, S.; Stenoien, D. L.; Paša-Tolić, L., High-Throughput Proteomics. Annual Review of Analytical Chemistry 2014, 7 (1), 427-454.

7. Wiśniewski, J. R.; Zougman, A.; Nagaraj, N.; Mann, M., Universal sample preparation method for proteome analysis. Nature Methods 2009, 6 (5), 359-362.

8. Speers, A. E.; Wu, C. C., Proteomics of Integral Membrane ProteinsTheory and Application. Chemical Reviews 2007, 107 (8), 3687-3714.

9. Loo, R. R.; Dales, N.; Andrews, P. C., Surfactant effects on protein structure examined by electrospray ionization mass spectrometry. Protein science : a publication of the Protein Society 1994, 3 (11), 1975-1983.

10. Waas, M.; Bhattacharya, S.; Chuppa, S.; Wu, X.; Jensen, D. R.; Omasits, U.; Wollscheid, B.; Volkman, B. F.; Noon, K. R.; Gundry, R. L., Combine and Conquer: Surfactants, Solvents, and Chaotropes for Robust Mass Spectrometry Based Analyses of Membrane Proteins. Analytical Chemistry 2014, 86 (3), 1551-1559.

11. Zougman, A.; Selby, P. J.; Banks, R. E., Suspension trapping (STrap) sample preparation method for bottom-up proteomics analysis. Proteomics 2014, 14 (9), 1006-1000.

12. Ludwig, K. R.; Schroll, M. M.; Hummon, A. B., Comparison of In-Solution, FASP, and S-Trap Based Digestion Methods for Bottom-Up Proteomic Studies. Journal of Proteome Research 2018, 17 (7), 2480-2490.

13. Brown, K. A.; Tucholski, T.; Eken, C.; Knott, S.; Zhu, Y.; Jin, S.; Ge, Y., High-Throughput Proteomics Enabled by a Photocleavable Surfactant. Angewandte Chemie International Edition 2020, 59 (22), 8406-8410.

14. Brown, K. A.; Chen, B.; Guardado-Alvarez, T. M.; Lin, Z.; Hwang, L.; Ayaz-Guner, S.; Jin, S.; Ge, Y., A photocleavable surfactant for top-down proteomics. Nature methods 2019, 16 (5), 417-420.

15. Knott, S. J.; Brown, K. A.; Josyer, H.; Carr, A.; Inman, D.; Jin, S.; Friedl, A.; Ponik, S. M.; Ge, Y., Photocleavable Surfactant-Enabled Extracellular Matrix Proteomics. Analytical Chemistry 2020, 92 (24), 15693-15698.

16. Batth, T. S.; Francavilla, C.; Olsen, J. V., Off-Line High-pH Reversed-Phase Fractionation for InDepth Phosphoproteomics. Journal of Proteome Research 2014, 13 (12), 6176-6186.

17. Bekker-Jensen, D. B.; Kelstrup, C. D.; Batth, T. S.; Larsen, S. C.; Haldrup, C.; Bramsen, J. B.; Sørensen, K. D.; Høyer, S.; Ørntoft, T. F.; Andersen, C. L.; Nielsen, M. L.; Olsen, J. V., An Optimized Shotgun Strategy for the Rapid Generation of Comprehensive Human Proteomes. Cell systems 2017, 4 (6), 587-599.e4.

18. Issaq, H. J.; Chan, K. C.; Janini, G. M.; Conrads, T. P.; Veenstra, T. D., Multidimensional separation of peptides for effective proteomic analysis. Journal of Chromatography B 2005, 817 (1), 3547.

19. Fournier, M. L.; Gilmore, J. M.; Martin-Brown, S. A.; Washburn, M. P., Multidimensional Separations-Based Shotgun Proteomics. Chemical Reviews 2007, 107 (8), 3654-3686. 
20. Gundry, R. L.; White, M. Y.; Murray, C. I.; Kane, L. A.; Fu, Q.; Stanley, B. A.; Van Eyk, J. E., Preparation of Proteins and Peptides for Mass Spectrometry Analysis in a Bottom-Up Proteomics Workflow. Current Protocols in Molecular Biology 2010, 90 (1), 10.25.1-10.25.23.

21. Wolters, D. A.; Washburn, M. P.; Yates, J. R., An Automated Multidimensional Protein Identification Technology for Shotgun Proteomics. Analytical Chemistry 2001, 73 (23), 5683-5690.

22. Gilar, M.; Olivova, P.; Daly, A. E.; Gebler, J. C., Two-dimensional separation of peptides using RP-RP-HPLC system with different $\mathrm{pH}$ in first and second separation dimensions. Journal of Separation Science 2005, 28 (14), 1694-1703.

23. Roca, L. S.; Gargano, A. F. G.; Schoenmakers, P. J., Development of comprehensive twodimensional low-flow liquid-chromatography setup coupled to high-resolution mass spectrometry for shotgun proteomics. Analytica Chimica Acta 2021, 1156, 338349.

24. Meier, F.; Brunner, A.-D.; Koch, S.; Koch, H.; Lubeck, M.; Krause, M.; Goedecke, N.; Decker, J.; Kosinski, T.; Park, M. A.; Bache, N.; Hoerning, O.; Cox, J.; Räther, O.; Mann, M., Online Parallel Accumulation-Serial Fragmentation (PASEF) with a Novel Trapped Ion Mobility Mass Spectrometer*. Molecular \& Cellular Proteomics 2018, 17 (12), 2534-2545.

25. Meier, F.; Beck, S.; Grassl, N.; Lubeck, M.; Park, M. A.; Raether, O.; Mann, M., Parallel Accumulation-Serial Fragmentation (PASEF): Multiplying Sequencing Speed and Sensitivity by Synchronized Scans in a Trapped Ion Mobility Device. Journal of Proteome Research 2015, 14 (12), 53785387.

26. Cox, J.; Mann, M., MaxQuant enables high peptide identification rates, individualized p.p.b.-range mass accuracies and proteome-wide protein quantification. Nat Biotechnol 2008, 26 (12), 1367-72.

27. Cox, J.; Neuhauser, N.; Michalski, A.; Scheltema, R. A.; Olsen, J. V.; Mann, M., Andromeda: A Peptide Search Engine Integrated into the MaxQuant Environment. Journal of Proteome Research 2011, 10 (4), 1794-1805.

28. Prianichnikov, N.; Koch, H.; Koch, S.; Lubeck, M.; Heilig, R.; Brehmer, S.; Fischer, R.; Cox, J., MaxQuant Software for Ion Mobility Enhanced Shotgun Proteomics*. Molecular \& Cellular Proteomics 2020, 19 (6), 1058-1069.

29. Tyanova, S.; Temu, T.; Sinitcyn, P.; Carlson, A.; Hein, M. Y.; Geiger, T.; Mann, M.; Cox, J., The Perseus computational platform for comprehensive analysis of (prote)omics data. Nature Methods 2016, $13(9), 731-740$.

30. Hulsen, T.; de Vlieg, J.; Alkema, W., BioVenn - a web application for the comparison and visualization of biological lists using area-proportional Venn diagrams. BMC Genomics 2008, 9 (1), 488.

31. Doll, S.; Dreßen, M.; Geyer, P. E.; Itzhak, D. N.; Braun, C.; Doppler, S. A.; Meier, F.; Deutsch, M.-A.; Lahm, H.; Lange, R.; Krane, M.; Mann, M., Region and cell-type resolved quantitative proteomic map of the human heart. Nature Communications 2017, 8 (1), 1469.

32. Linscheid, N.; Santos, A.; Poulsen, P. C.; Mills, R. W.; Calloe, K.; Leurs, U.; Ye, J. Z.; Stolte, C.; Thomsen, M. B.; Bentzen, B. H.; Lundegaard, P. R.; Olesen, M. S.; Jensen, L. J.; Olsen, J. V.; Lundby, A., Quantitative proteome comparison of human hearts with those of model organisms. PLOS Biology 2021, 19 (4), e3001144.

33. Lam, M. P. Y.; Wang, D.; Lau, E.; Liem, D. A.; Kim, A. K.; Ng, D. C. M.; Liang, X.; Bleakley, B. J.; Liu, C.; Tabaraki, J. D.; Cadeiras, M.; Wang, Y.; Deng, M. C.; Ping, P., Protein kinetic signatures of the remodeling heart following isoproterenol stimulation. The Journal of Clinical Investigation 2014, 124 (4), 1734-1744.

34. Kim, M.-S.; Pinto, S. M.; Getnet, D.; Nirujogi, R. S.; Manda, S. S.; Chaerkady, R.; Madugundu, A. K.; Kelkar, D. S.; Isserlin, R.; Jain, S.; Thomas, J. K.; Muthusamy, B.; Leal-Rojas, P.; Kumar, P.; Sahasrabuddhe, N. A.; Balakrishnan, L.; Advani, J.; George, B.; Renuse, S.; Selvan, L. D. N.; Patil, A. H.; Nanjappa, V.; Radhakrishnan, A.; Prasad, S.; Subbannayya, T.; Raju, R.; Kumar, M.; Sreenivasamurthy, S. K.; Marimuthu, A.; Sathe, G. J.; Chavan, S.; Datta, K. K.; Subbannayya, Y.; Sahu, A.; Yelamanchi, S. D.; Jayaram, S.; Rajagopalan, P.; Sharma, J.; Murthy, K. R.; Syed, N.; Goel, R.; Khan, A. A.; Ahmad, S.; Dey, G.; Mudgal, K.; Chatterjee, A.; Huang, T.-C.; Zhong, J.; Wu, X.; Shaw, P. G.; Freed, D.; Zahari, M. S.; Mukherjee, K. K.; Shankar, S.; Mahadevan, A.; Lam, H.; Mitchell, C. J.; Shankar, S. K.; Satishchandra, P.; 
Schroeder, J. T.; Sirdeshmukh, R.; Maitra, A.; Leach, S. D.; Drake, C. G.; Halushka, M. K.; Prasad, T. S. K.; Hruban, R. H.; Kerr, C. L.; Bader, G. D.; Iacobuzio-Donahue, C. A.; Gowda, H.; Pandey, A., A draft map of the human proteome. Nature 2014, 509 (7502), 575-581.

35. Linscheid, N.; Poulsen, P. C.; Pedersen, I. D.; Gregers, E.; Svendsen, J. H.; Olesen, M. S.; Olsen, J. V.; Delmar, M.; Lundby, A., Quantitative Proteomics of Human Heart Samples Collected In Vivo Reveal the Remodeled Protein Landscape of Dilated Left Atrium Without Atrial Fibrillation. Molecular \& cellular proteomics : MCP 2020, 19 (7), 1132-1144.

36. Hu, H.-L.; Kang, Y.; Zeng, Y.; Zhang, M.; Liao, Q.; Rong, M.-Q.; Zhang, Q.; Lai, R., Regionresolved proteomics profiling of monkey heart. Journal of Cellular Physiology 2019, 234 (8), 13720-13734. 37. Liu, S.; Xia, Y.; Liu, X.; Wang, Y.; Chen, Z.; Xie, J.; Qian, J.; Shen, H.; Yang, P., In-depth proteomic profiling of left ventricular tissues in human end-stage dilated cardiomyopathy. Oncotarget; Vol 8, No 292017.

38. Kulak, N. A.; Geyer, P. E.; Mann, M., Loss-less Nano-fractionator for High Sensitivity, High Coverage Proteomics*. Molecular \& Cellular Proteomics 2017, 16 (4), 694-705. 
Figures

A. 1. Cardiac Tissue Cutting (Scalable down to $1 \mathrm{mg}$ )
2. Tissue Homogenization in $0.2 \%$ Azo Surfactant
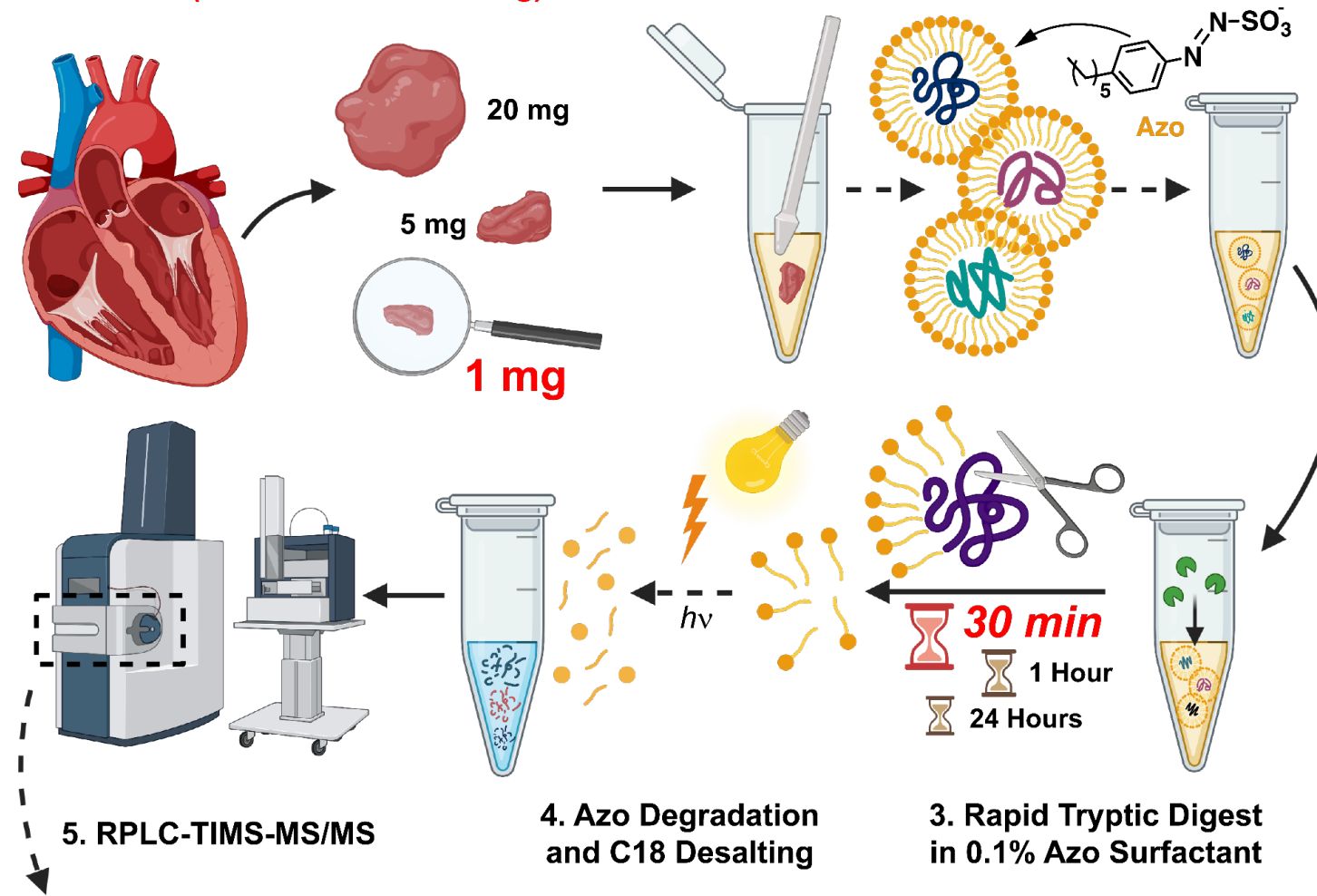

3. Rapid Tryptic Digest in $0.1 \%$ Azo Surfactant
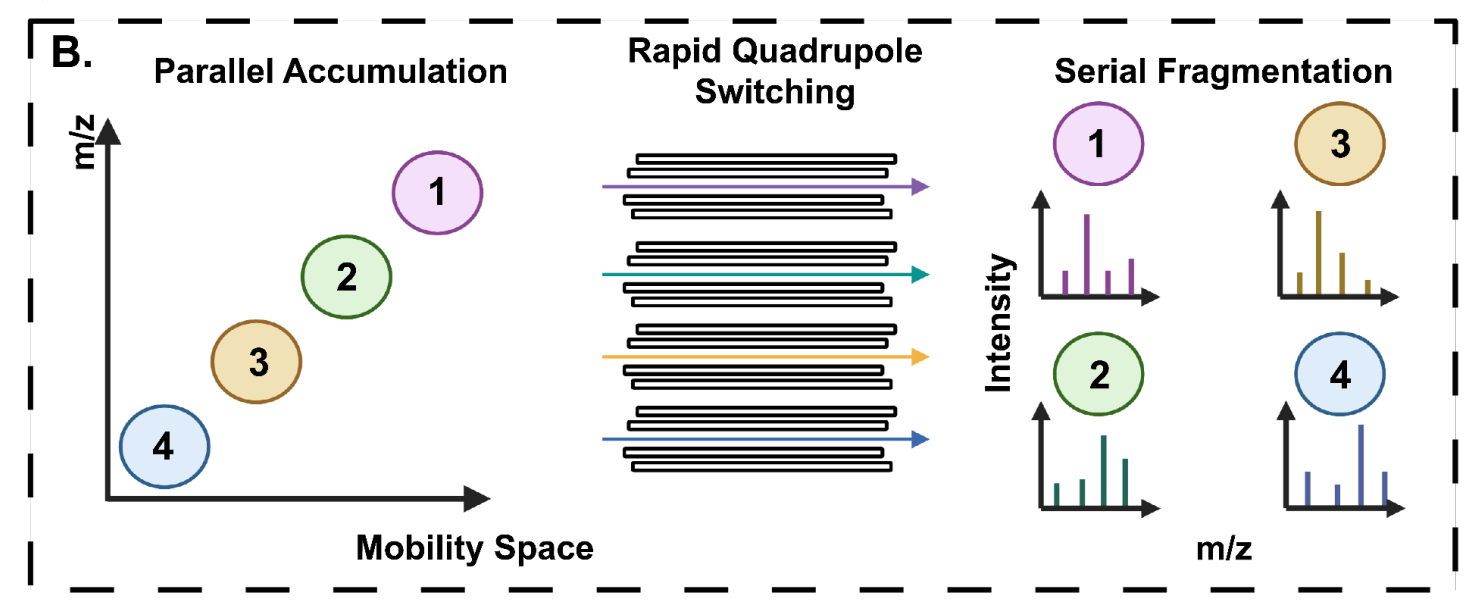

Figure 1. Global bottom-up proteomics workflow using Azo and PASEF on the timsTOF Pro. (A) Sample preparation, including (1) tissue cutting (scalable down to $1 \mathrm{mg}$ of tissue), (2) tissue homogenization in Azo, a photocleavable surfactant, (3) rapid tryptic digest (as quick as 30 min), and (4) Azo degradation and C18 desalting prior to (5) RPLC-TIMS-MS/MS. (B) Schematic of PASEF on the timsTOF Pro, demonstrating parallel accumulation of ions, rapid quadrupole mass filter switching, and serial ion fragmentation. 
A. Digest Time (h)

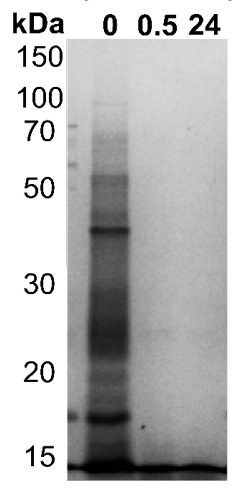

D.

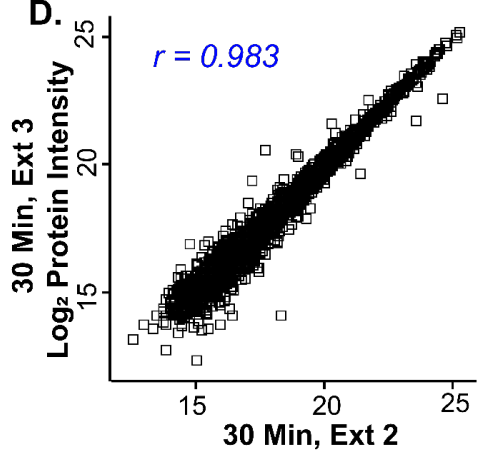

G.

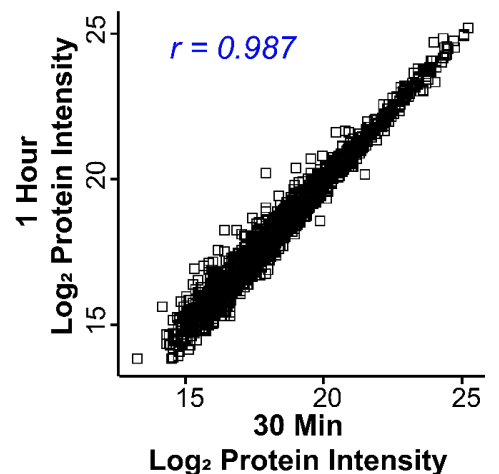

B. Unique Protein Groups Identified

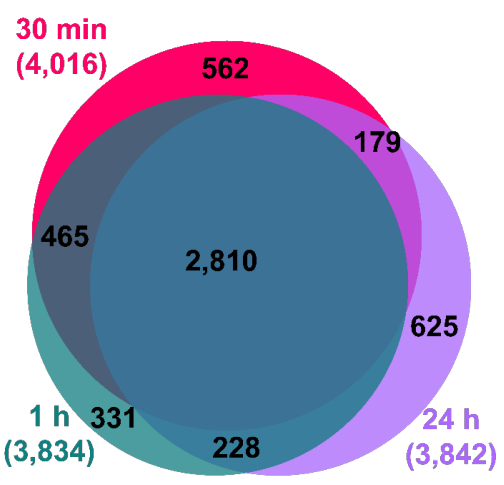

C. Unique Peptides Identified $30 \mathrm{~min}$

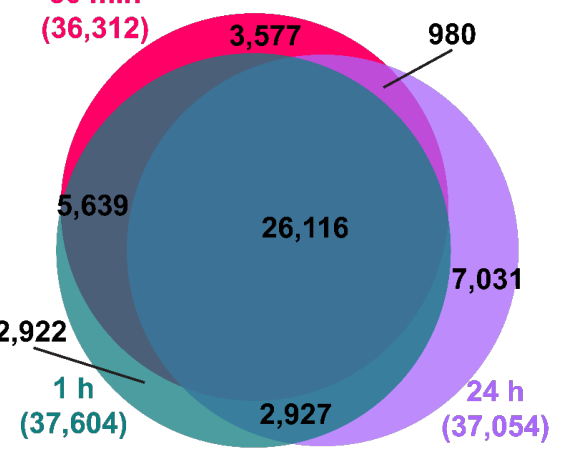

E.

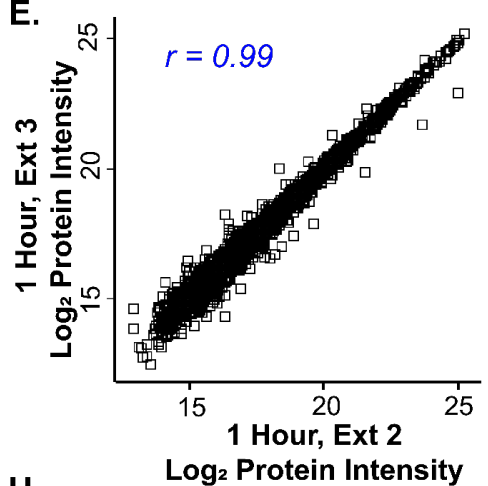

H.

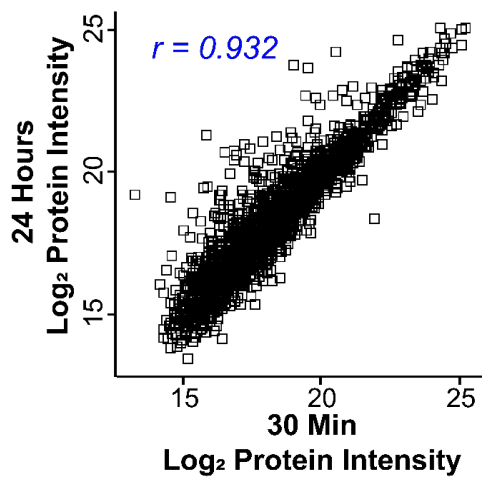

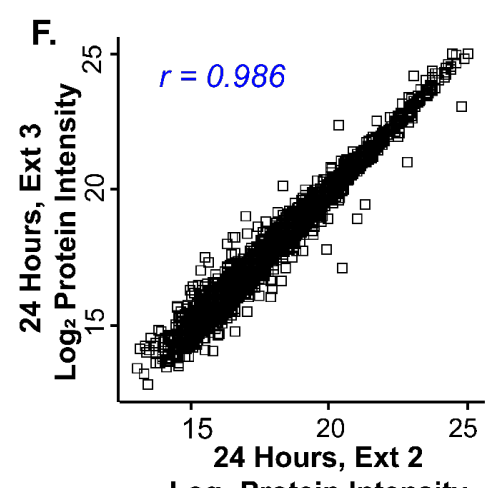

I. $\quad \log _{2}$ Protein Intensity

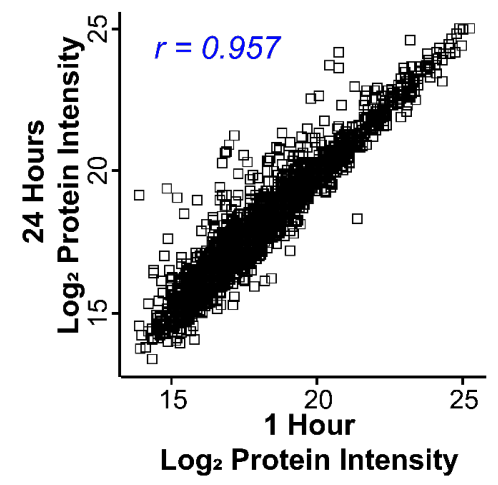

Figure 2. Azo promotes rapid and reproducible tryptic digestion for quantitative proteomics. (A) SDS-PAGE showing tryptic digestion efficiency in $0.1 \%$ Azo after $0.5 \mathrm{~h}$ and $24 \mathrm{~h}$ of digestion. (B and C) Venn diagrams illustrating overlap in unique protein groups (B), and unique peptides (C) identified by MaxQuant between $30 \mathrm{~min}, 1 \mathrm{~h}$, and $24 \mathrm{~h}$ tryptic digestions $(\mathrm{n}=3$ replicates for each group). (D-F) Scatterplots of $\log _{2}$ LFQ protein intensities showing high reproducibility between replicates from $30 \mathrm{~min}(\mathbf{D}), 1 \mathrm{~h} \mathrm{(E),} \mathrm{and} 24 \mathrm{~h} \mathrm{(F)} \mathrm{tryptic} \mathrm{digestions.} \mathrm{Pearson} \mathrm{correlation}$ coefficients are shown in the top left corner of each panel. (G-I) Scatterplots of $\log _{2}$ LFQ protein intensities showing high reproducibility between averaged replicates from 30 min digestions plotted against $1 \mathrm{~h}$ digestions (G), 30 min digestions plotted against $24 \mathrm{~h}$ digestions $(\mathbf{H})$, and $1 \mathrm{~h}$ digestions plotted against $24 \mathrm{~h}$ digestions (I) with Pearson correlation coefficients shown in the top left corner of each panel ( $\mathrm{n}=3$ replicates for each group). 
A. Unique Protein Groups Identified

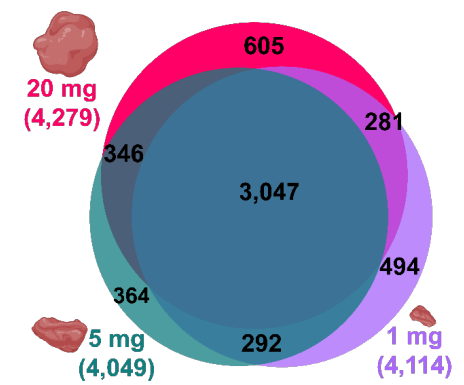

C.

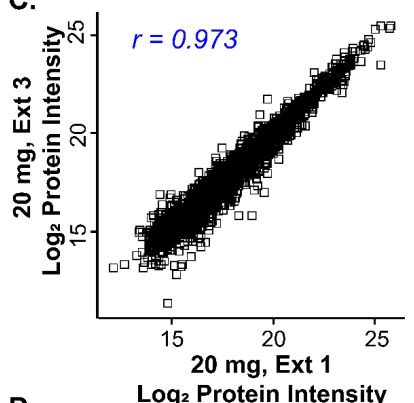

D.

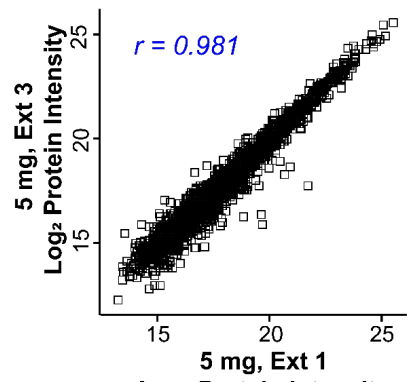

E. $\quad \log _{2}$ Protein Intensity

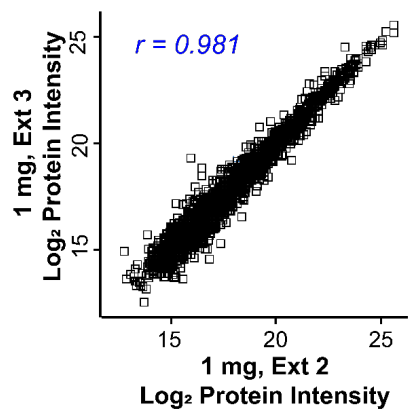

B. Unique Peptides Identified

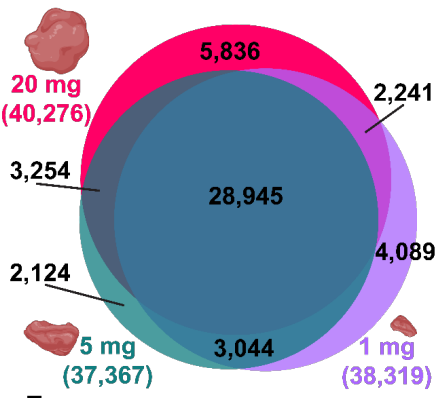

F.

G.
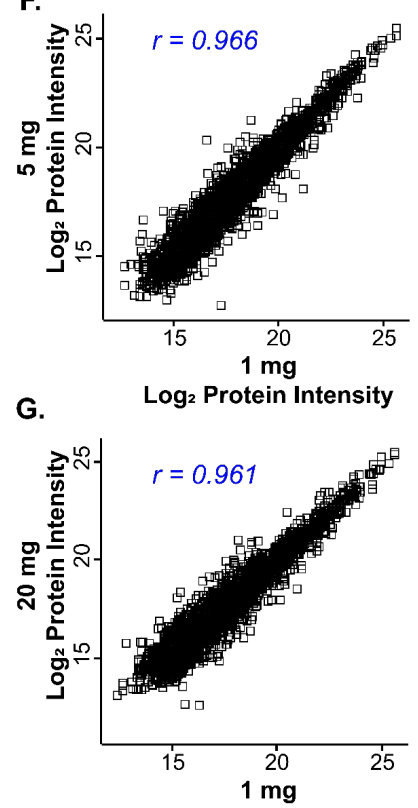

H.

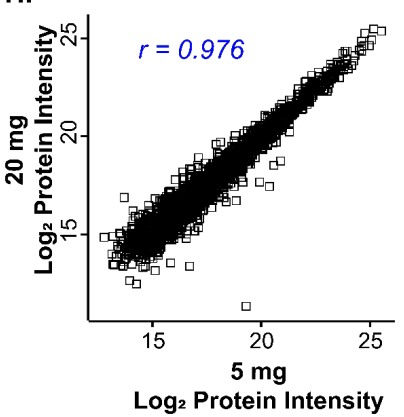

Figure 3. Reproducible protein identification and quantitation from small amounts of tissue. (A and B) Venn diagrams illustrating overlap in unique protein groups (A), and unique peptides (B) identified by MaxQuant between $20 \mathrm{mg}, 5 \mathrm{mg}$, and $1 \mathrm{mg}$ of tissue ( $\mathrm{n}=3$ replicates for each group). (D-F). Scatterplots of $\log _{2}$ LFQ protein intensities showing high reproducibility between replicates from $20 \mathrm{mg}$ (D), $5 \mathrm{mg}$ (E), and $1 \mathrm{mg}$ (F) tissue extractions. Pearson correlation coefficients are shown in the top left corner of each panel. (G-I) Scatterplots of $\log _{2}$ LFQ protein intensities showing high reproducibility between averaged replicates from $1 \mathrm{mg}$ extractions plotted against $5 \mathrm{mg}$ extractions (G), $1 \mathrm{mg}$ extractions plotted against $20 \mathrm{mg}$ extractions (H), and $5 \mathrm{mg}$ extractions plotted against $20 \mathrm{mg}$ extractions (I) with Pearson correlation coefficients shown in the top left corner of each panel ( $\mathrm{n}=3$ replicates for each group). 
A.

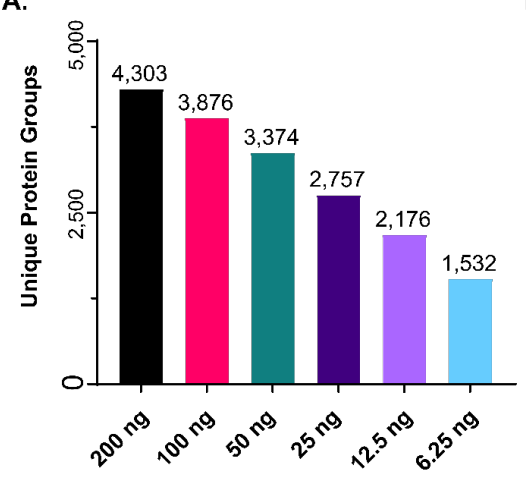

C.

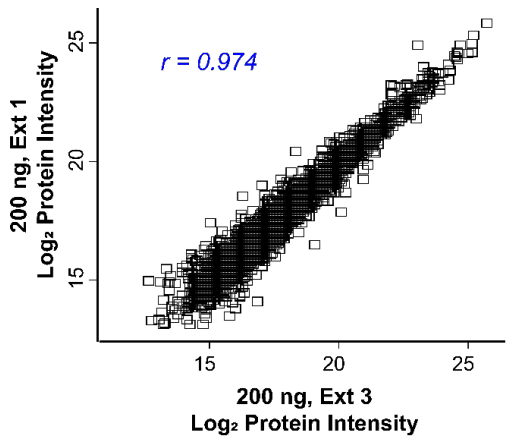

B.

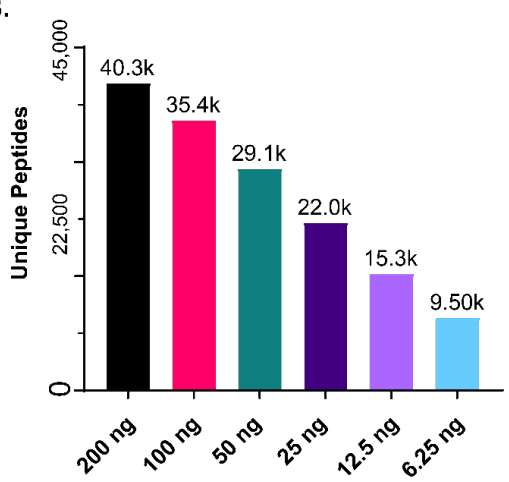

D.

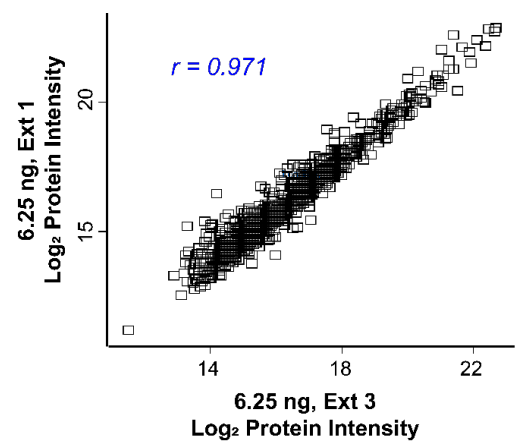

Figure 4. LFQ analysis with high reproducibility from low sample loading amounts on the timsTOF Pro. (A and B). Bar charts showing the total number of unique protein groups (A), and unique peptides (B), identified by MaxQuant from injections of 200, 100, 50, 25, 12.5, and 6.25 ng of digested peptide ( $\mathrm{n}=3$ for each group), respectively. Total number of identifications are displayed above each bar. (C and D). Scatterplots of $\log _{2}$ LFQ protein intensities showing high reproducibility between replicates from $200 \mathrm{ng}$ (C) and $6.25 \mathrm{ng}$ (D) injections. Pearson correlation coefficients are shown in the top left corner of each panel. 
bioRxiv preprint doi: https://doi.org/10.1101/2021.05.24.445470; this version posted May 26, 2021. The copyright holder for this preprint (which

was not certified by peer review) is the author/funder, who has granted bioRxiv a license to display the preprint in perpetuity. It is made available under aCC-BY-NC-ND 4.0 International license.

\section{TOC}

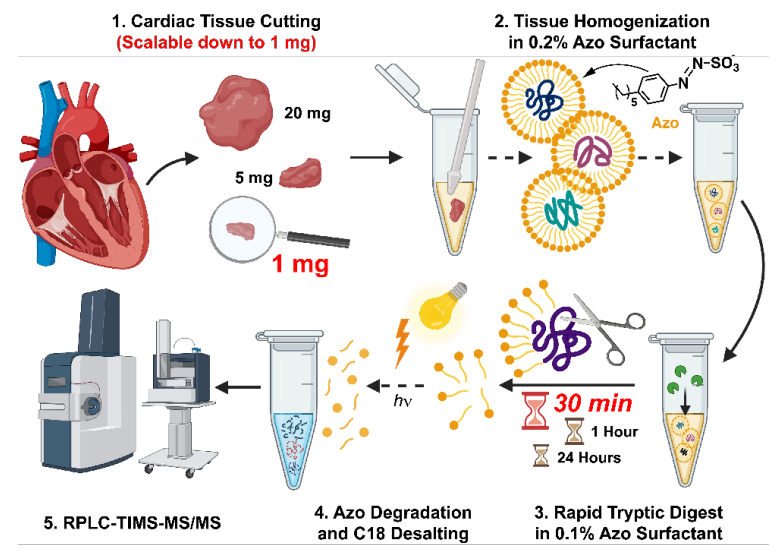

